05

\title{
Приближение Бете для двумерной спин-псевдоспиновой системы
}

\author{
() Ю.Д. Панов, А.С. Москвин, В.А. Улитко, А.А. Чиков \\ Уральский фредеральный университет, \\ Екатеринбург, Россия \\ E-mail: yuri.panov@urfu.ru \\ Поступила в Редакцию 15 апреля 2019 г. \\ В окончательной редакции 22 апреля 2019 г. \\ Принята к публикации 24 апреля 2019 г. \\ Рассмотрена двумерная спин-псевдоспиновая модель, которая обобщает разбавленную антиферромагнит- \\ ную модель Изинга с заряженными немагнитными примесями на случай двух типов зарядов. Аналитические \\ результаты в приближении Бете сравниваются с результатами численного моделирования классическим \\ методом Монте-Карло при различных параметрах.
}

Ключевые слова: модель Изинга, приближение Бете, купраты.

DOI: 10.21883 /FTT.2019.09.48110.17N

\section{1. Введение}

В течение последних пятнадцати лет в физике купратов активно исследуется проблема зарядового упорядочения [1-5] и взаимное влияние спинового и зарядового упорядочения в купратах [6-10]. Ранее [11-14] для рассмотрения конкуренции спинового и зарядового упорядочений нами была предложена упрощенная статическая двумерная спин-псевдоспиновая модель для $\mathrm{CuO}_{2}$ плоскости купрата, в которой наряду с $\mathrm{CuO}_{4}^{6-}$ центрами, обладающими спином $\frac{1}{2}$, рассматриваются взаимодействующие $\mathrm{CuO}_{4}^{5-}$ и $\mathrm{CuO}_{4}^{7-}$-центры со спином 0 в основном состоянии. Многоэлектронные валентные состояния $\mathrm{CuO}_{4}^{7-; 6-; 5-}$ (соответствующие, формально, состояниям ионов меди $\left.\mathrm{Cu}^{1+; 2+; 3+}\right)$ описываются как компоненты псевдоспинового триплета $S=1$ с $M_{S}=-1,0,+1$ соответственно, что позволяет использовать псевдоспиновый формализм $[15,16]$. В рамках этого подхода гамильтониан статической спин-псевдоспиновой модели имеет вид

$$
\mathscr{H}=\Delta \sum_{i} S_{z i}^{2}+V \sum_{\langle i j\rangle} S_{z i} S_{z j}+J s^{2} \sum_{\langle i j\rangle} \sigma_{z i} \sigma_{z j}-\mu \sum_{i} S_{z i},
$$

где $S_{z i}$ это $z$-компонента псевдоспина $S=1$ на узле $i, \sigma_{z i}=P_{0 i} s_{z i} / s-$ нормированная $z$-компонента спина $s=1 / 2$, умноженная на проектор $P_{0 i}=1-S_{z i}^{2}$ на состояние с $M_{S}=0$. Параметры $\Delta=U / 2$ и $V>0$ соответствуют зарядовым корреляциям на узле и между узлами решетки, $J$ - обменное взаимодействие Изинга. Химический потенциал $\mu$ позволяет учесть условие $n N=\sum\left\langle S_{z i}\right\rangle=\mathrm{const}$, где $n-$ плотность заряда в $\mathrm{CuO}_{2}$-плоскости, отсчитываемого от родительского состава. Суммирование идет по узлам двумерной квадратной решетки, $\langle i j\rangle$ означает ближайших соседей.

Спин-псевдоспиновая модель обобщает двумерную разбавленную антиферромагнитную (AFM) модель Изинга с заряженными примесями. При $\Delta \rightarrow-\infty$ она сводится к модели Изинга для спина $S=\frac{1}{2}$ с фиксированной намагниченностью. При $\Delta>0$ результаты можно сравнить с результатами моделей Блюма-Капеля $[17,18]$ или моделью Блюма-Эмери-Гриффитса [19]. Модель Изинга с подвижными заряженными примесями также была рассмотрена в работе [20].

Фазовые диаграммы основного состояния спинпсевдоспиновой системы в приближении среднего поля были рассмотрены ранее [11,12]. Было показано, что в системе в основном состоянии возможны 5 фаз в двух различных пределах. В пределе слабого обмена, при $J s^{2}<V$, все фазы (COI, COII, COII, FIM) соответствуют зарядовому упорядочению (CO) типа шахматной доски при средней плотности заряда $n$. В то время, как в фазе COI нет спиновых центров $\left(\mathrm{Cu}^{2+}\right)$, фазы COII и COIII разбавляются невзаимодействующими спиновыми центрами, распределенными только в одной подрешетке. За счет этого формально имеет место ферримагнитное спиновое упорядочение, что является результатом приближения среднего поля. Численные расчеты классическим методом МонтеКарло $(M C)$ показывают в этих случаях при низких температурах парамагнитный отклик. Фаза FIM также формально ферримагнитна. В этом случае спиновое AFM-упорядочение разбавляется невзаимодействующими зарядовыми центрами $\left(\mathrm{Cu}^{1+3+}\right)$, распределенными только в одной подрешетке. Граница между фазой COI и фазами COII и COIII соответствует линии $\Delta=0$, между фазой COIII и фазами COII и FIM - линии $|n|=1 / 2$. Граница между фазами COII и FIM определяется выражением $\Delta=2\left(V-J s^{2}\right)$. В пределе сильного обмена, при $J s^{2}>V$, реализуются только фазы COІ и AFM, граница между которыми определяется выражением $\Delta=2\left(V-J s^{2}(1-|n|)\right)$. Согласно предположению метода среднего поля для основного состояния, в фазах COI и AFM зарядовые центры равномерно распределены в обеих подрешетках. Однако результаты численного моделирования $[21,22]$ показывают, что в AFM-фазе при 
сильном обмене наблюдается фазовое расслоение (PS) на псевдоспиновую (зарядовую) и магнитную (спиновую) подсистемы, при котором AFM-матрица выталкивает заряженные немагнитные примеси, минимизируя поверхностную энергию. Далее, в работе [12] было рассмотрено влияние неоднородностей потенциала и магнитной доменной структуры на процесс фазового расслоения, а в работе [14] были исследованы температурные фазовые диаграммы и термодинамические характеристики спин-псевдоспиновой системы в приближении среднего поля и получено аналитическое выражение для температуры фазового расслоения в рамках построения Максвелла. В настоящей работе мы исследуем вопрос об устойчивости результатов среднего поля по отношения к частичному учету близкодействующих спиновых и псевдоспиновых корреляций в рамках приближения Бете. Нас интересуют количественные и возможные качественные различия термодинамических характеристик в зависимости от увеличения размера кластера Бете для различных участков фазовой диаграммы, и, в частности, вблизи границ между разными типами упорядочения, где их конкуренция существенна.

Статья организована следующим образом. Мы формулируем различные варианты приближения Бете для двумерной спин-псевдоспиновой системы в разделе 2. В разделе 3 мы сравниваем аналитические результаты для критической температуры и теплоемкости с результатами приближения среднего поля [14] и численного моделирования классическим методом $M C$. Раздел 4 посвящен выводам.

\section{2. Приближение Бете для двумерной спин-псевдоспиновой системы}

Стандартным образом мы вводим на квадратной решетке две подрешетки $\alpha=A, B$, и записываем гамильтониан нулевого приближения в виде $\mathscr{H}_{0}=\sum_{c} \mathscr{H}_{c}$, где $\mathscr{H}_{c}$ - гамильтониан отдельного кластера Бете. Различные варианты кластеров покрывающих всю решетку, которые рассмотрены в этой работе, приведены на рис. 1 . Выбор кластера 1 соответствует обычному приближению среднего поля [14]. Выражение для гамильтониана кластера запишем в виде

$$
\begin{aligned}
\beta \mathscr{H}_{c} & =\delta \sum_{i}^{c} S_{z i}^{2}+v \sum_{\langle i j\rangle}^{c} S_{z i} S_{z j}+j \sum_{\langle i j\rangle}^{c} \sigma_{z i} \sigma_{z j}-\xi_{z} \sum_{i}^{c} S_{z i} \\
& -\xi \sum_{i}^{o}(-1)^{\alpha_{i}} S_{z i}-\gamma_{z} \sum_{i}^{o} \sigma_{z i}-\gamma \sum_{i}^{o}(-1)^{\alpha_{i}} \sigma_{z i},
\end{aligned}
$$

где $\beta=1 / k_{B} T$ (и далее мы полагаем $k_{B}=1$ ), $\delta=\beta \Delta$, $v=\beta V, j=\beta J s^{2},(-1)^{A, B}= \pm 1$. Обозначение $\sum^{c}$ указывает на суммирование по всем узлам кластера, граничным и внутренним, $\sum^{o}$ предполагает суммирование только по граничным узлам. Для кластеров 1, 2, 4 внутренних узлов нет, поэтому $\sum^{c}$ и $\sum^{o}$ совпадают; в

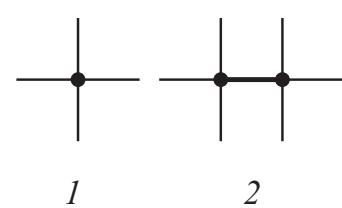

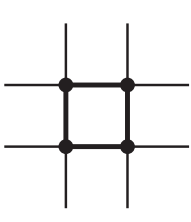

4

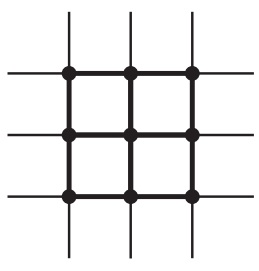

9
Рис. 1. Различный выбор кластеров Бете. Кластер 1 соответствует обычному приближению среднего поля.

кластере 9 есть внутренний узел. Молекулярные поля $\xi_{z}$, $\xi$ и $\gamma_{z}, \gamma$ являются вариационными параметрами и учитывают, соответственно, заряд-зарядовое и обменное взаимодействие узлов кластера Бете с внешним окружением. За счет короткодействующего характера взаимодействий в гамильтониане (1), в эти слагаемые необходимо включать только граничные узлы кластера Бете. Необходимость введения двух типов полей $\left(\gamma_{z}\right.$ и $\gamma$ для спинов, $\xi_{z}$ и $\xi$ для псевдоспинов) обусловлена антиферромагнитным характером спинового и псевдоспинового взаимодействия. При наличии внутренних узлов в кластере Бете появляется возможность введения дополнительных вариационных параметров - молекулярных полей, действующих на внутренние узлы [23]. В настоящей работе мы выбираем вариант, когда поле $\xi_{z}$ действует одинаково на граничные и на внутренние узлы кластера, а остальные молекулярные поля для внутренних узлов равны нулю. В связи с этим, выражение (2) для $\mathscr{H}_{c}$ содержит $\sum^{c}$ для поля $\xi_{z}$. Это позволяет нам наиболее просто учесть условие постоянства заряда, поскольку при таком выборе поле $\xi_{z}$ сопряжено суммарной $z$-компоненте псевдоспина.

В рамках исходного полуфеноменологического подхода Бете вариационные параметры определяются из некоторого дополнительного условия, например, из условия согласования значений параметров порядка для внутренних и граничных узлов. В настоящей работе мы применяем вариационный подход, основанный на неравенстве Боголюбова [24], который, как известно [23], дает лучшие результаты в смысле оценки значения свободной энергии.

Мы используем неравенство Боголюбова для оценки большого потенциала $\Omega(\mathscr{H}): \Omega(\mathscr{H}) \leq \Omega=\Omega\left(\mathscr{H}_{0}\right)$ $+\left\langle\mathscr{H}-\mathscr{H}_{0}\right\rangle$, и для оценки свободной энергии системы в расчете на один узел решетки, $f=\Omega / N+\mu n$, получаем выражение

$$
\begin{aligned}
\beta f= & -\frac{1}{N_{c}} \ln Z_{c}+\frac{c_{1}}{2}\left[v\left(n^{2}-a_{0}^{2}\right)+j\left(m_{o}^{2}-l_{o}^{2}\right)\right] \\
& +\xi_{z} n+c_{2}\left[\xi a_{o}+\gamma_{z} m_{o}+\gamma l_{o}\right] .
\end{aligned}
$$

Здесь $Z_{c}-$ статистическая сумма кластера. В случае кластеров 22 и 4 на рис. $1 \quad Z_{c}=\exp \left[-\beta \mathscr{H}_{c}\right]$, для кластеров 1 и 9 выражение имеет вид 
Параметры в формулах (3)-(7) для различных кластеров Бете

\begin{tabular}{c|c|c|c|c}
\hline Кластер Бете & $N_{c}$ & $N_{o}$ & $c_{1}$ & $c_{2}$ \\
\hline 1 & 2 & 2 & 4 & 1 \\
2 & 2 & 2 & 3 & 1 \\
4 & 4 & 4 & 2 & 1 \\
9 & 18 & 16 & $4 / 3$ & $8 / 9$
\end{tabular}

$Z_{c}=\exp \left[-\beta\left(\mathscr{H}_{c A}+\mathscr{H}_{c B}\right)\right]$, где $\mathscr{H}_{c \alpha}-$ гамильтониан (2) с центральным узлом на подрешетке $\alpha$. Плотность заряда $n$ и параметры порядка $a_{o}, m_{o}, l_{o}$ выражаются через молекулярные поля стандартным образом

$$
\begin{gathered}
n=\frac{\left\langle S_{z}\right\rangle_{A}+\left\langle S_{z}\right\rangle_{B}}{2}=\frac{1}{N_{c}} \frac{\partial \ln Z_{c}}{\partial \xi_{z}}, \\
\alpha_{o}=\frac{\left\langle S_{z o}\right\rangle_{A}-\left\langle S_{z o}\right\rangle_{B}}{2}=\frac{1}{N_{o}} \frac{\partial \ln Z_{c}}{\partial \xi} \\
m=\frac{\left\langle\sigma_{z o}\right\rangle_{A}+\left\langle\sigma_{z o}\right\rangle_{B}}{2}=\frac{1}{N_{o}} \frac{\partial \ln Z_{c}}{\partial \gamma_{z}} \\
l_{o}=\frac{\left\langle\sigma_{z o}\right\rangle_{A}-\left\langle\sigma_{z o}\right\rangle_{B}}{2}=\frac{1}{N_{o}} \frac{\partial \ln Z_{c}}{\partial \gamma}
\end{gathered}
$$

Индекс $o$ указывает на принадлежность к границе кластера. Параметры $N_{c}, N_{o}, c_{1}, c_{2}$ приведены в таблице. Мы не приводим явные аналитические выражения для статистической суммы кластера и параметров порядка ввиду их громоздкости: так, если для кластеров 1 и 2 число экспонент с различными показателями в статистической сумме составляет 16 , то для кластера $4-99$, для кластера $9-15703$. Для работы с этими выражениями мы использовали пакет Wolfram Mathematica.

Минимизация свободной энергии $f$ приводит к системе уравнений для определения значений параметров порядка $a_{o}, m_{o}, l_{o}$ при заданных $T$ и $n$ :

$$
\begin{gathered}
-c_{1} v a_{o}+c_{2} \xi=0, \quad c_{1} j m_{o}+c_{2} \gamma_{z}=0, \\
-c_{1} j l_{o}+c_{2} \gamma=0 .
\end{gathered}
$$

Анализ полученных решений показывает, что при $J>0$ в отсутствии внешнего магнитного поля минимумы с $m_{o} \neq 0\left(\gamma_{z} \neq 0\right)$ не реализуются. Как и в приближении среднего поля [14], в зависимости от соотношения параметров $\Delta, V, J$ при заданном $n$ с понижением температуры происходит фазовый переход из неупорядоченной (NO) в зарядово-упорядоченную (CO) фазу с $a_{o} \neq 0$ или антиферромагнитную (AFM) фазу с $l_{o} \neq 0$. Температуры фазового перехода можно найти из условий потери устойчивости минимума NO фазы по соответствующему параметру порядка

$$
T_{C O}=\left.\frac{c_{1} V}{c_{2} N_{o} Z_{c}} \frac{\partial^{2} Z_{c}}{\partial \xi^{2}}\right|_{N O}, T_{A F M}=-\left.\frac{c_{1} J s^{2}}{c_{2} N_{o} Z_{c}} \frac{\partial^{2} Z_{c}}{\partial \gamma^{2}}\right|_{N O} .
$$

Эти уравнения относительно параметров $\xi_{z}$ и $T_{C O}$ или $T_{A F M}$ необходимо дополнить уравнением $n=$ const.

\section{3. Обсуждение результатов}

Результаты для критической температуры и теплоемкости, полученные в различных вариантах приближения Бете для двумерной спин-псевдоспиновой системы мы сравниваем с результатами численного моделирования этой системы классическим методом $M C$. Мы использовали алгоритм Метрополиса на плоской квадратной решетке $64 \times 64$ с периодическими граничными условиями для системы с гамильтонианом (1) без последнего слагаемого, пропорционального химическому потенциалу $\mu$, так как условие постоянства заряда, $\sum S_{z i}=\mathrm{const}$, в нашей программе соблюдалось точно: на каждом шаге мы изменяли состояние произвольно выбранной пары узлов решетки $a$ и $b$, требуя сохранения величины $S_{z a}+S_{z b}$. Мы реализовали высокопроизводительные параллельные вычисления на видеокартах NVIDIA, что позволило нам проводить расчет для 64 копий системы одновременно. Критическая температура упорядочения или фазового разделения определялась по максимуму теплоемкости. Для нашей системы $64 \times 64$ с гамильтонианом типа Изинга это дает ошибку не более $2 \%$ по сравнению с критической температурой, полученной нами для нескольких наборов параметров методом конечно-размерного скейлинга.

На рис. 2 приведены зависимости от $\Delta$ критических температур, определенных из уравнений (7) для кластеров $1,2,4,9$ (линии) и найденных методом $M C$ (кружки) для случая сильного обмена $(n=0.1, J=1.0, V=0.1)$. Вертикальная пунктирная линия при $\Delta_{M C} / J=-0.295$ показывает границу между фазой $\mathrm{CO}$ и фазами AFM

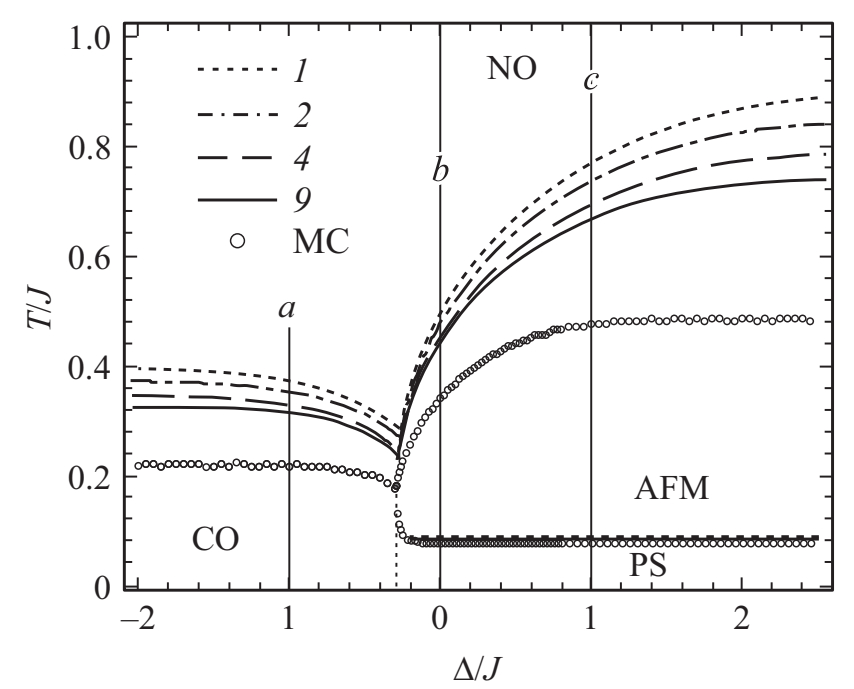

Рис. 2. Фазовая диаграмма спин-псевдоспиновой модели в случае сильного обмена: $n=0.1, J=1.0, V / J=0.1$. Кружки соответствуют критическим температурам, полученным методом $M C$. Линии 1,2, 4, 9 соответствуют различным приближениям Бете (см. таблицу). Вертикальные линии $a, b, c$ показывают сечения, при которых вычислялась теплоемкость на рис. $3,4,5$. Вертикальная пунктирная линия при $\Delta / J=-0.295$ показывает границу между фазой CO и фазами AFM и PS. 
и $\mathrm{PS}$, определенную по результатам $M C$. Это значение несколько ниже, чем критическое значение $\Delta$, которое соответствует границе между фазами основного состояния СОI и АFМ в случае сильного обмена: $\Delta / J=-0.27$. Различие связано, прежде всего, с представлением о равномерном распределении центров с $S_{z}= \pm 1$ (в зависимости от знака $n$ ) в AFM-фазе основного состояния, что приводит [13] к выражению для удельной энергии в виде $\varepsilon_{A F M}(n)=|n| \Delta-2 J s^{2}(1-|n|)^{2}+2 n^{2} V$. Если предположить в основном состоянии макроскопическое разделение фаз, где одна фаза с удельной энергией $\varepsilon_{C}(1)=2 V+\Delta$ представляет собой центры с $S_{z}=1$ или $S_{z}=-1$, в зависимости от знака $n$, а вторая фаза это AFM с $n=0$, и, аналогично работе [14], использовать для основного состояния построение Максвелла: $|n| \varepsilon_{C}(1)+(1-|n|) \varepsilon_{A F M}(0)=\varepsilon_{A F M}(n)$, то получим критическое значение $\Delta=2\left(V-J s^{2}\right)$. Интересно отметить, что это выражение совпадает с тем, которое определяет границу между фазами COII и FIM в случае слабого обмена. При рассматриваемых параметрах получим $\Delta / J=-0.3$.

Как показывает рис. 2, с увеличением размера кластера результаты приближения Бете для критической температуры приближаются к результатам $M C$. Это наиболее заметно в предельных случаях (при $\Delta \rightarrow \pm \infty$ ), однако вблизи точки фрустрации, т.е. в области значений $\Delta$, где происходит смена типа упорядочения с $\mathrm{CO}$ на AFM, наблюдается более медленная сходимость.

Используя построение Максвелла для свободной энергии

$$
|n| f_{C}(1)+(1-|n|) f(0)=f(n),
$$

и полагая $f_{C}(1)=\varepsilon_{C}(1)$, можно определить температуру фазового расслоения $T_{P S}$ в AFM-фазе в случае сильного обмена. Расчеты в приближении Бете по формуле (8) показывают, что $T_{P S}$ для кластера 1 (приближение среднего поля), для которой получено аналитическое выражение [14] в виде

$$
T_{P S}=2\left(V-J S^{2}\right) \frac{|n|(1-|n|)}{|n| \ln |n|+(1-|n|) \ln (1-|n|)},
$$

отличается от $T_{P S}$ для кластера 9 примерно на $0.015 \mathrm{~J}$, из-за чего на рис. 2 соответствующие линии для разных выборов кластера Бете практически сливаются.

На рис. 3,4 и 5 приведены температурные зависимости удельной теплоемкости, полученные в различных приближениях Бете и рассчитанные с помощью метода $M C$ при $n=0.1, J=1.0, V=0.1$ (случай сильного обмена), и значениях $\Delta / J=-1.0,0.0,1.0$ соответственно. Во всех случаях положение пика теплоемкости, соответствующего фазовогому переходу, его высота и высокотемпературная часть зависимости теплоемкости с увеличением размера кластера Бете последовательно приближаются к численным результатам, однако характерный для приближения среднего поля конечный скачок теплоемкости при фазовом переходе остается.

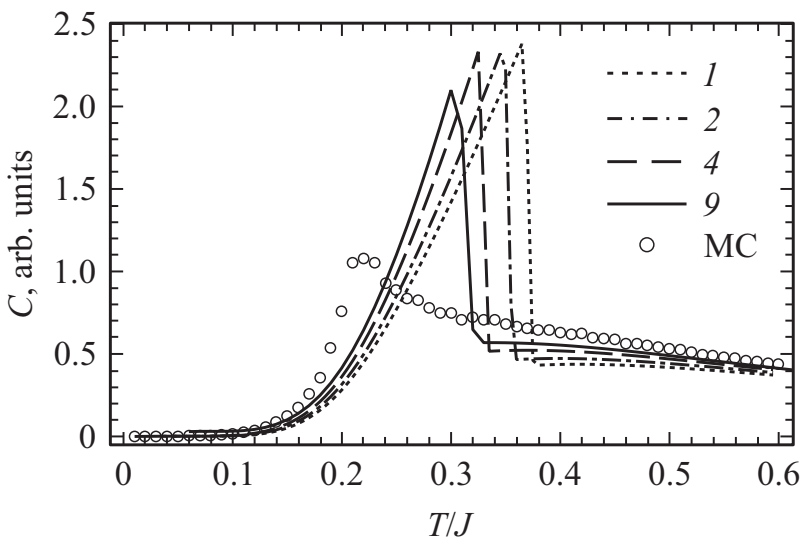

Рис. 3. Значения удельной теплоемкости, полученные в приближении Бете $1,2,4,9$ (см. таблицу) и методом $M C$ (кружки) при $n=0.1, J=1.0, V / J=0.1, \Delta / J=-1$ (линия $a$ на рис. 2).

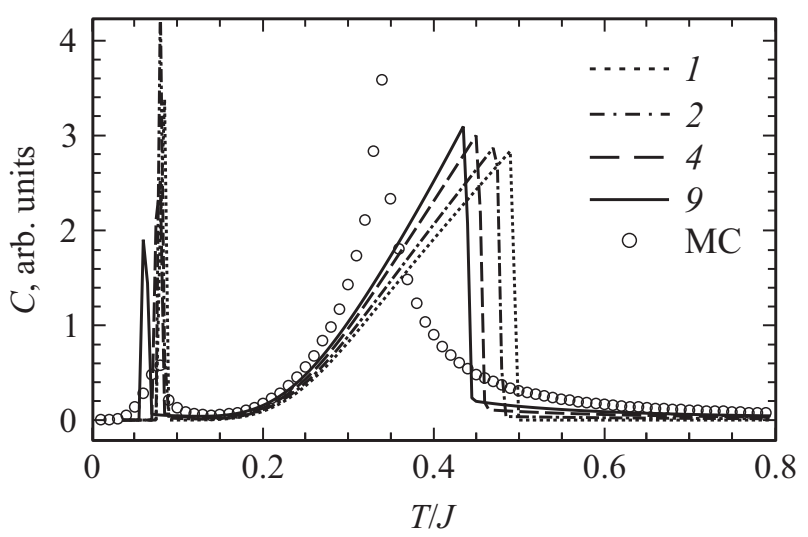

Рис. 4. Значения удельной теплоемкости, полученные в приближении Бете $1,2,4,9$ (см. таблицу) и методом $M C$ (кружки) при $n=0.1, J=1.0, V / J=0.1, \Delta / J=0$ (линия $b$ на рис. 2).

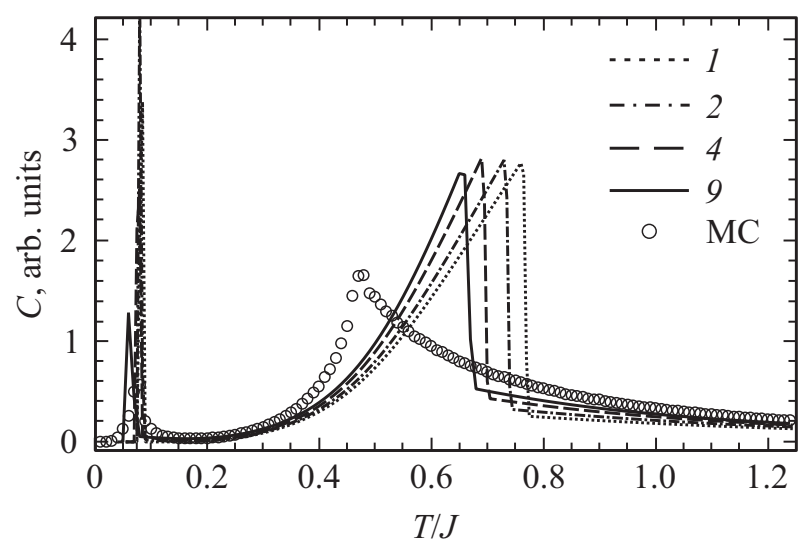

Рис. 5. Значения удельной теплоемкости, полученные в приближении Бете $1,2,4,9$ (см. таблицу) и методом $M C$ (кружки) при $n=0.1, J=1.0, V / J=0.1, \Delta / J=1$ (линия $c$ на рис. 2).

Аналогичные расчеты были проведены и для случая слабого обмена при $n=0.1, J=1.0, V=1.0$. На рис. 6 приведены зависимости от $\Delta$ критических температур, 


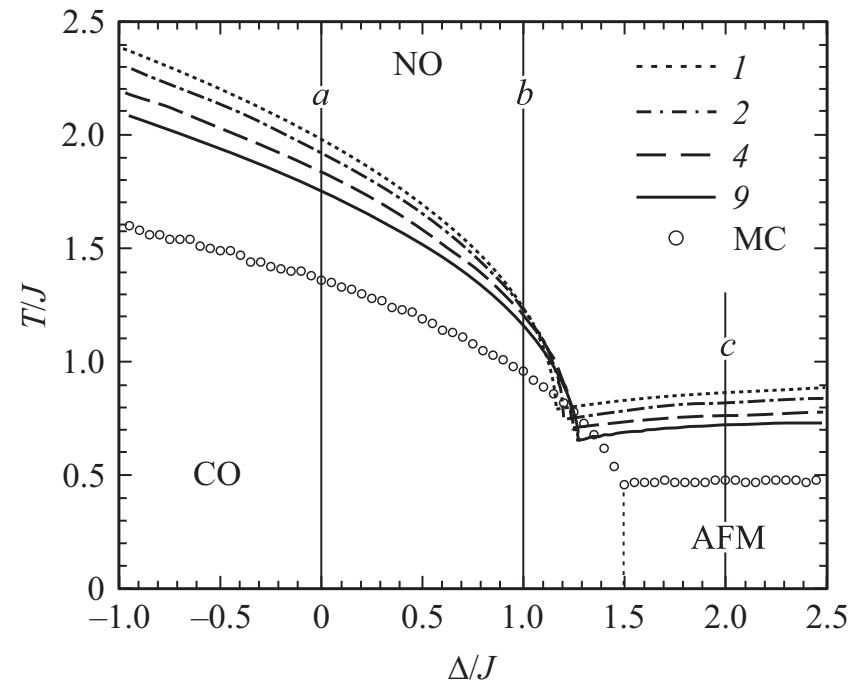

Рис. 6. Фазовая диаграмма спин-псевдоспиновой модели в случае слабого обмена: $n=0.1, J=1.0, V / J=1$. Кружки соответствуют критическим температурам, полученным методом $M C$. Линии $1,2,4,9$ соответствуют различным приближениям Бете (см. таблицу). Вертикальные линии $a, b, c$ показывают сечения, при которых вычислялась теплоемкость на рис. $7,8,9$. Вертикальная пунктирная линия при $\Delta / J=1.48$ показывает границу между фазами CO и AFM.

полученных по формулам (7) в приближениях Бете с кластерами $1,2,4,9$ (линии) и найденных методом $M C$ (кружки). В этом случае приближение среднего поля дает оценку точки фрустрации $\Delta_{1} / J=1.20$, что значительно отличается от значения, полученного в численном моделировании $\Delta_{M C} / J=1.48$. Последовательность приближений Бете постепенно улучшает оценку приближения среднего поля, приводя в нашем случае к $\Delta_{9} / J=1.26$.

На рис. 7, 8 и 9 изображены температурные зависимости удельной теплоемкости для случая слабого обмена при $n=0.1, J=1.0, V=1.0$ и значениях $\Delta / J=0.0$, $1.0,2.0$. Отметим качественное различие результатов вблизи точки фрустрации, при $\Delta / J=1.0$, и вдали от нее, при $\Delta / J=0.0$ и $\Delta / J=2.0$. Положение пика теплоемкости с увеличением кластера Бете при $\Delta / J=1.0$ изменяется не так значительно, как при $\Delta / J=0.0$ и $\Delta / J=2.0$, однако его величина для кластера Бете 9 существенно меньше, чем в приближении среднего поля. При $\Delta / J=0.0$ и $\Delta / J=2.0$, напротив, величина пика теплоемкости с увеличением кластера Бете меняется слабо. Такое поведение в целом можно связать с наличием двух типов флуктуаций вблизи точки фрустрации в спиновой и псевдоспиновой подсистеме одновременно, тогда как с удалением от точки фрустрации, при $\Delta \rightarrow \pm \infty$, одна из подсистем с понижением температуры „вымерзает“ еще до точки фазового перехода. Частичный учет флуктуаций при увеличении кластера Бете приводит к более реалистичной оценке скачка теплоемкости вблизи точки фрустрации.
В целом, для нашей системы результаты приближения Бете качественно совпадают с результатами среднего поля. Точный учет части взаимодействий для кластеров 2, 4, 9 приводит к постепенному количественному

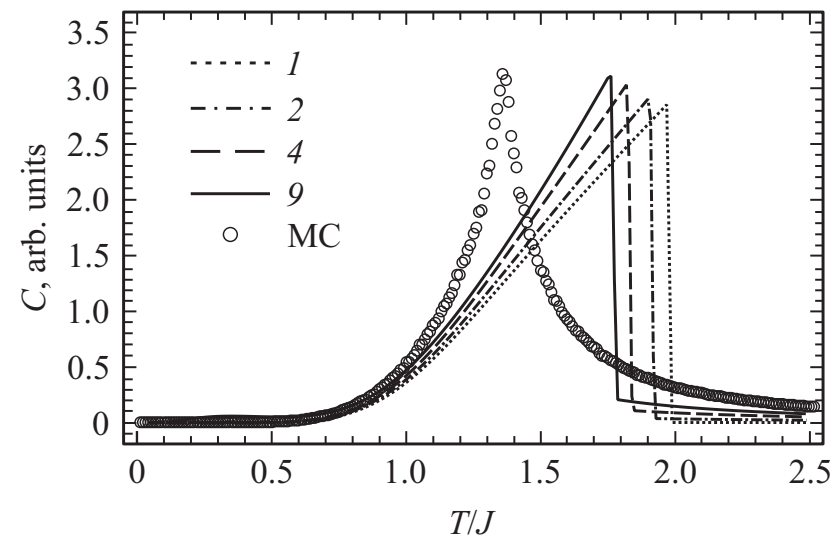

Рис. 7. Значения удельной теплоемкости, полученные в приближении Бете 1, 2, 4,9 (см. таблицу) и методом $M C$ (кружки) при $n=0.1, J=1.0, V / J=1, \Delta / J=0$ (линия $a$ на рис. 6).

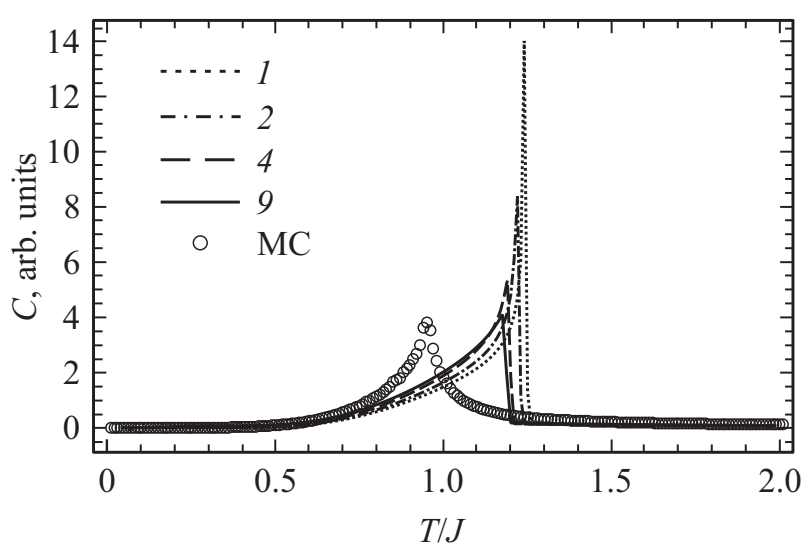

Рис. 8. Значения удельной теплоемкости, полученные в приближении Бете $1,2,4,9$ (см. таблицу) и методом $M C$ (кружки) при $n=0.1, J=1.0, V / J=1, \Delta / J=1$ (линия $b$ на рис. 6 ).

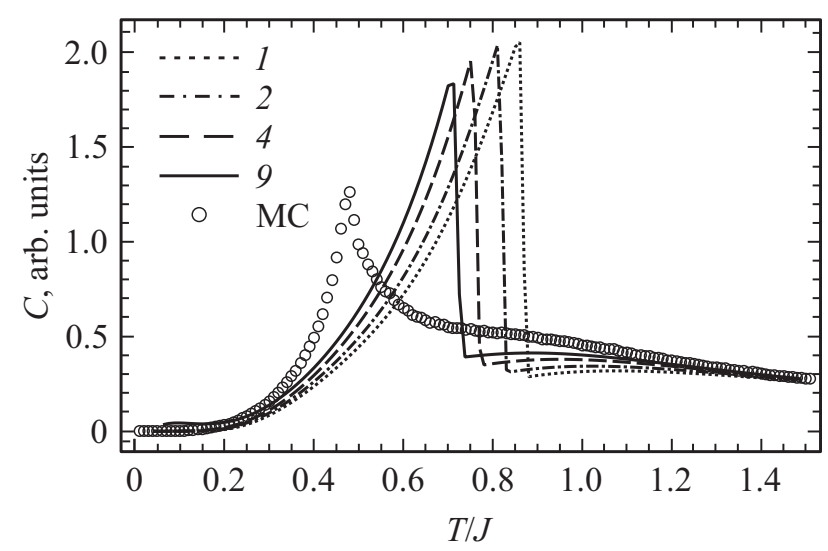

Рис. 9. Значения удельной теплоемкости, полученные в приближении Бете $1,2,4,9$ (см. таблицу) и методом $M C$ (кружки) при $n=0.1, J=1.0, V / J=1, \Delta / J=2$ (линия $c$ на рис. 6 ). 
уточнению значений критических температур, при этом наибольший эффект заметен вдали от точки фрустрации. Увеличение размера кластера Бете означает более полный учет флуктуаций ближнего порядка, что улучшает результаты для теплоемкости в высокотемпературной области и приводит к существенно меньшему, по сравнению с приближением среднего поля, значению скачка теплоемкости вблизи точки фрустрации в случае слабого обмена. Наблюдаемая качественная и количественная близость результатов приближения Бете и приближения среднего поля в нашей системе связана с рассматриваемым типом взаимодействий это короткодействующие взаимодействия типа Изинга. В этом случае отсутствуют квантовые эффекты, связанные с недиагональными матричными элементами в гамильтониане. Также очевидно, что дальние корреляции по-прежнему описываются приближенно, поэтому характер критического поведения остается прежним [23]. Приближение Бете, будучи развитием метода среднего поля, не может также детально описать процесс фазового расслоения в АFМ-фазе в пределе сильного обмена или явления перколяции, которые, по-видимому, существенны в пределе слабого обмена в AFM-фазе при переходе от малых концентраций заряда к большим.

\section{4. Заключение}

Приближение Бете с увеличением размера кластера позволяет последовательно уточнить результаты приближения среднего поля для значений критических температур фазового перехода и фазового расслоения двумерной спин-псевдоспиновой системы при различных параметрах системы. Наибольший эффект достигается вдали от точки фрустрации. Частичный учет флуктуаций при увеличении кластера Бете приближает аналитические результаты для теплоемкости к результатам численного моделирования методом Монте-Карло в неупорядоченной фазе и приводит к более реалистичной оценке скачка теплоемкости, особенно вблизи точки фрустрации. При этом с увеличением кластера Бете типичный для приближения среднего поля конечный скачок теплоемкости сохраняется.

\section{Финансирование работы}

Работа выполнена при поддержке Программы 211 Правительства Российской Федерации, соглашение 02.А03.21.0006 и проектов № 2277 и № 5719 Министерства образования и науки Российской Федерации и гранта РФФИ № 18-32-00837_18

\section{Конфликт интересов}

Авторы заявляют, что у них нет конфликта интересов.

\section{Список литературы}

[1] T. Wu, H. Mayaffre, S. Krämer, M. Horvatić, C. Berthier, W.N. Hardy, R. Liang, D.A. Bonn, M.-H. Julien. Nature 477, 191 (2011)

[2] J. Chang, E. Blackburn, A.T. Holmes, N.B. Christensen, J. Larsen, J. Mesot, R. Liang, D.A. Bonn, W.N. Hardy, A. Watenphul, M.V. Zimmermann, E.M. Forgan, S.M. Hayden. Nature Phys. 8, 871 (2012).

[3] E.H. da Silva Neto, P. Aynajian, A. Frano, R. Comin, E. Schierle, E. Weschke, A. Gyenis, J. Wen, J. Schneeloch, Z. Xu, S. Ono, G. Gu, M. Le Tacon, A. Yazdani. Science 343, 393 (2014).

[4] R. Comin, A. Damascelli. Ann. Rev. Condens. Matter Phys. 7, 369 (2016).

[5] F. Laliberté, M. Frachet, S. Benhabib, B. Borgnic, T. Loew, J. Porras, M. Le Tacon, B. Keimer, S. Wiedmann, C. Proust, D. LeBoeuf. npj Quantum Mater. 3, 11 (2018).

[6] P. Abbamonte, A. Rusydi, S. Smadici, G.D. Gu, G.A. Sawatzky, D.L. Feng. Nature Phys., 1, 155 (2005).

[7] E. Berg, E. Fradkin, S.A. Kivelson, J.M. Tranquada. New J. Phys. 11, 115004 (2009).

[8] E. Fradkin, S.A. Kivelson. Nature Phys. 8, 864 (2012).

[9] G. Drachuck, E. Razzoli, G. Bazalitski, A. Kanigel, C. Niedermayer, M. Shi, A. Keren. Nature Commun. 5, 3390 (2014).

[10] O. Cyr-Choinière, G. Grissonnanche, S. Badoux, J. Day, D.A. Bonn, W.N. Hardy, R. Liang, N. Doiron-Leyraud, L. Taillefer. Phys. Rev. B 92, 224502 (2015).

[11] Yu.D. Panov, A.S. Moskvin, A.A. Chikov, I.L. Avvakumov. J. Superconduct. Nov. Magn. 29, 1077 (2016).

[12] Yu.D. Panov, A.S. Moskvin, A.A. Chikov, K.S. Budrin. J. Low Temp. Phys. 187, 646 (2017).

[13] Yu.D. Panov, K.S. Budrin, A.A. Chikov, A.S. Moskvin. Письма в ЖЭТФ 106, 242 (2017).

[14] Ю.Д. Панов, В.А. Улитко, К.С. Будрин, Д.Н. Ясинская, А.А. Чиков. ФТТ 61, 822 (2019).

[15] A.S. Moskvin. J. Phys.: Condens. Matter 25, 085601 (2013).

[16] A.S. Moskvin. Phys. Rev. B 84, 075116 (2011).

[17] M. Blume. Phys. Rev. 141, 517 (1966).

[18] H.W. Capel. Physica 32, 966 (1966).

[19] M. Blume, V.J. Emery, R.B. Griffiths. Phys. Rev. A 4, 1071 (1971).

[20] С.В. Семкин, В.П. Смагин. ФТТ 57, 926 (2015).

[21] A. Chikov, Y. Panov, A. Moskvin, K. Budrin. Acta Phys. Polon. A 133 3, 432-434 (2018).

[22] K.S. Budrin, Y.D. Panov, A.S. Moskvin, A.A. Chikov. EPJWeb Conf. 185, 11006 (2018).

[23] И.А. Квасников. Термодинамика и статистическая физика. Т. 2, Теория равновесных систем: Статистическая физика. Едиториал УРСС, М. (2002). 432 с.

[24] A.L. Kuzemsky. Int. J. Mod. Phys. B 29, 1530010 (2015).

Редактор Ю.Э. Китаев 